Revue des patrimoines

27 | 2015

Le cheval et ses patrimoines (2e partie)

\title{
Le cheval, de la fosse au musée : l'exemple du Centre historique minier
}

\section{Virginie Debrabant}

\section{(2) OpenEdition}

Journals

\section{Édition électronique}

URL : http://journals.openedition.org/insitu/12121

DOI : 10.4000/insitu. 12121

ISSN : 1630-7305

\section{Éditeur}

Ministère de la culture

Référence électronique

Virginie Debrabant, «Le cheval, de la fosse au musée : I'exemple du Centre historique minier », In Situ [En ligne], 27 | 2015, mis en ligne le 02 novembre 2015, consulté le 19 avril 2019. URL : http:// journals.openedition.org/insitu/12121; DOI : 10.4000/insitu.12121

Ce document a été généré automatiquement le 19 avril 2019

\section{(c) $($ i) $(9)$}

In Situ Revues des patrimoines est mis à disposition selon les termes de la licence Creative Commons Attribution - Pas d'Utilisation Commerciale - Pas de Modification 4.0 International. 


\title{
Le cheval, de la fosse au musée: l'exemple du Centre historique minier
}

\author{
Virginie Debrabant
}

1 Avant même la fin de l'exploitation, l'entreprise des Houillères du bassin du Nord-Pas-deCalais décida de conserver sa mémoire ${ }^{1}$. C'est à l'initiative d'un homme, Alexis Destruys, alors secrétaire général du Bassin minier, que fut conservée la fosse Delloye à Lewarde, dès 1973. L'objectif était en effet de fonder un conservatoire de la mémoire rassemblant sur un site minier objets, archives, machines pour apporter aux générations suivantes le témoignage des trois siècles d'activité de l'exploitation minière.

2 En 1982, l'Association du Centre historique minier fut créée pour gérer le projet. Elle rassemblait au sein de son conseil d'administration les représentants de l'entreprise mais aussi ceux de l'État et des collectivités territoriales - la région, les départements du Nord et du Pas-de-Calais, la commune de Lewarde - et également des représentants des cinq syndicats de mineurs. Puis en 1984, ce fut l'ouverture au public avec dès cette année plus de 17000 visiteurs. En 1990, date de la fin de l'activité charbonnière dans la région, le Centre historique minier passa sous le contrôle des collectivités régionales. Une nouvelle équipe arriva à la direction du Centre, dont le directeur général, André Dubuc qui impulsa une nouvelle politique et développa les axes culturels, pédagogiques et touristiques.

3 L'exposition permanente «Le cheval et la mine » illustre les orientations patrimoniales, culturelles et scientifiques que le Centre historique minier a prises depuis sa création.

\section{De la fosse au Centre historique minier : des choix patrimoniaux}

4 Le bassin minier du Nord-Pas de Calais s'étend de Valenciennes à Bruay-la-Buissière, englobant les régions de Douai (Nord), Lens et Béthune (Pas-de-Calais) sur une longueur d'environ $120 \mathrm{~km}$ mais sur une faible largeur qui n'excède jamais $12 \mathrm{~km}$, soit environ $1 / 12$ e de la superficie totale du Nord-Pas-de-Calais. L'exploitation du charbon dans cette 
région s'est faite au cours de trois siècles, de 1720 à 1990, par des compagnies privées dans un premier temps. En 1946, la nationalisation des mines crée les Charbonnages de France et les Houillères de bassin, dont les HBNPC (les Houillères du bassin du Nord-Pasde-Calais). Au total, ce sont deux milliards de tonnes de charbon qui furent extraites de ce bassin minier, la plus forte activité se situant autour des années 1930 à 1960. En 1947, 250000 personnes en moyenne étaient employées à l'extraction d'environ 30 millions de tonnes par an.

\section{La fosse Delloye}

5 La Compagnie des mines d'Aniche entreprit en 1911 à Lewarde le fonçage ou creusement d'un premier puits destiné à servir de retour d'air à la fosse Vuillemin toute proche. Interrompus par la Première Guerre mondiale, les travaux de creusement reprirent au début des années 1920 dans le but de créer un site d'extraction à part entière. Le puits $n^{\circ} 1$ fut approfondi à cette époque jusqu'à 350 mètres; un second puits fut creusé à partir de 1927. Le bâtiment regroupant les bureaux, les bains-douches, la lampisterie et l'infirmerie ou " salle des blessés » a été construit de 1927 à 1928. Les salles des bains-douches ont été achevées et peintes en 1929. L'extraction démarra en 1931. La profondeur maximum d'extraction atteignait 479 mètres au puits numéro 1 dans les années 1960. En 1931, elle n'employait que 165 personnes et 18634 tonnes de charbon étaient extraites; le record fut atteint en 1963, avec plus de 440000 tonnes. Au plus fort de son activité, en 1947, elle comptait 1019 personnes qui travaillaient en alternance, réparties en plusieurs postes. Par la suite, le nombre total d'employés a été de 800 personnes en moyenne. Plus de huit millions de tonnes de charbon en ont été extraites de 1931 jusqu'au 13 juillet 1971. Dans le contexte de fin programmée des mines, la fosse Delloye fit partie des premières à être fermée, étant donné la pauvreté du gisement, tant en quantité qu'en qualité, et les conditions géologiques défavorables : les veines de charbon y sont en effet étroites, rares sont celles qui atteignent un mètre d'épaisseur.

\section{Le choix de la fosse Delloye}

6 Lorsque les HBNPC décidèrent de créer le Centre historique minier le 6 novembre 1973, le choix de la fosse Delloye à Lewarde s'imposa rapidement. L'exploitation s'était achevée deux ans auparavant et le site était en instance de démantèlement.

Plusieurs éléments concoururent à ce choix : construite à la fin des années 1920, elle est représentative de l'architecture industrielle minière dans sa période florissante, celle de la reconstitution des régions occupées après la Première Guerre mondiale. Par ailleurs, le style développé par la Compagnie d'Aniche est remarquable par son élégance, en particulier les toitures des chevalements de type oriental. Le site de la fosse Delloye est également complet (fig. 1); l'ensemble des installations a été conservé: bâtiment d'accueil et locaux de préparation au travail (bureaux, salles de bains, lampisterie), les passerelles du personnel et de mise à stock, les puits et leurs machines d'extraction, les salles des compresseurs, la scierie et bien sûr l'écurie du jour. Le carreau de la fosse Delloye regroupe donc $7000 \mathrm{~m}^{2}$ de bâtiments industriels sur un site d'un peu plus de 8 hectares. 


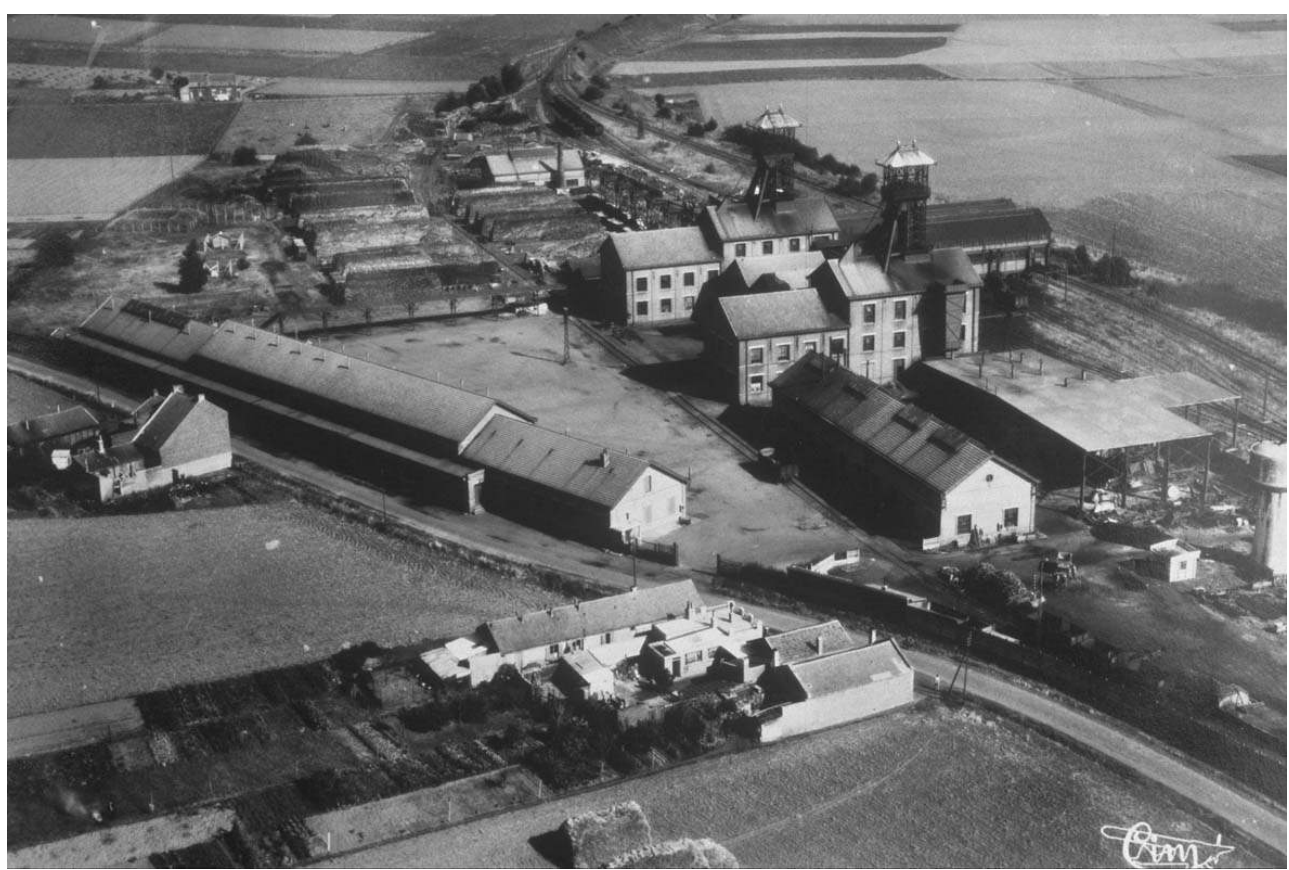

LEWARDE (NORD). VUE AÉRIENNE DE LA FOSSE DELLOYE.

(c) HBNPC, CENTRE HISTORIQUE MINIER, ANNÉES 1950.

Enfin, sa situation géographique est un atout supplémentaire : la fosse se situe à Lewarde, à 8 kilomètres de Douai, où se trouve le siège des HBNPC. Elle a aussi une position centrale dans le bassin minier, à proximité du réseau autoroutier.

\section{L'implantation du Centre historique minier}

9 La première phase d'implantation fut engagée par les HBNPC: des travaux de conservation et de remise en état des bâtiments furent entrepris. Dans le même temps, en 1976, les objectifs du Centre furent précisés : il s'agissait, comme l'indique une note du service de documentation des Houillères, de "conserver un siège de la période où le charbon était la principale source d'énergie, de collecter matériels et outils, rassembler les documents relatifs à la vie industrielle, sociale et artistique de la région ». Les années 1980 voient les premiers travaux sur le site: aménagement d'une salle d'exposition, d'équipements nécessaires à l'accueil du public, d'un auditorium. Le point central est celui du compromis permanent: compromis entre l'efficacité des dispositifs mis en place et respect de l'authenticité de la fosse de mine. Ainsi, la verrière des machines construite en 1986 entre les deux bâtiments des machines d'extraction répond à ces deux critères: le verre permet non seulement de comprendre l'ajout architectural mais aussi de lire par transparence les bâtiments de la fosse (fig. 2). 


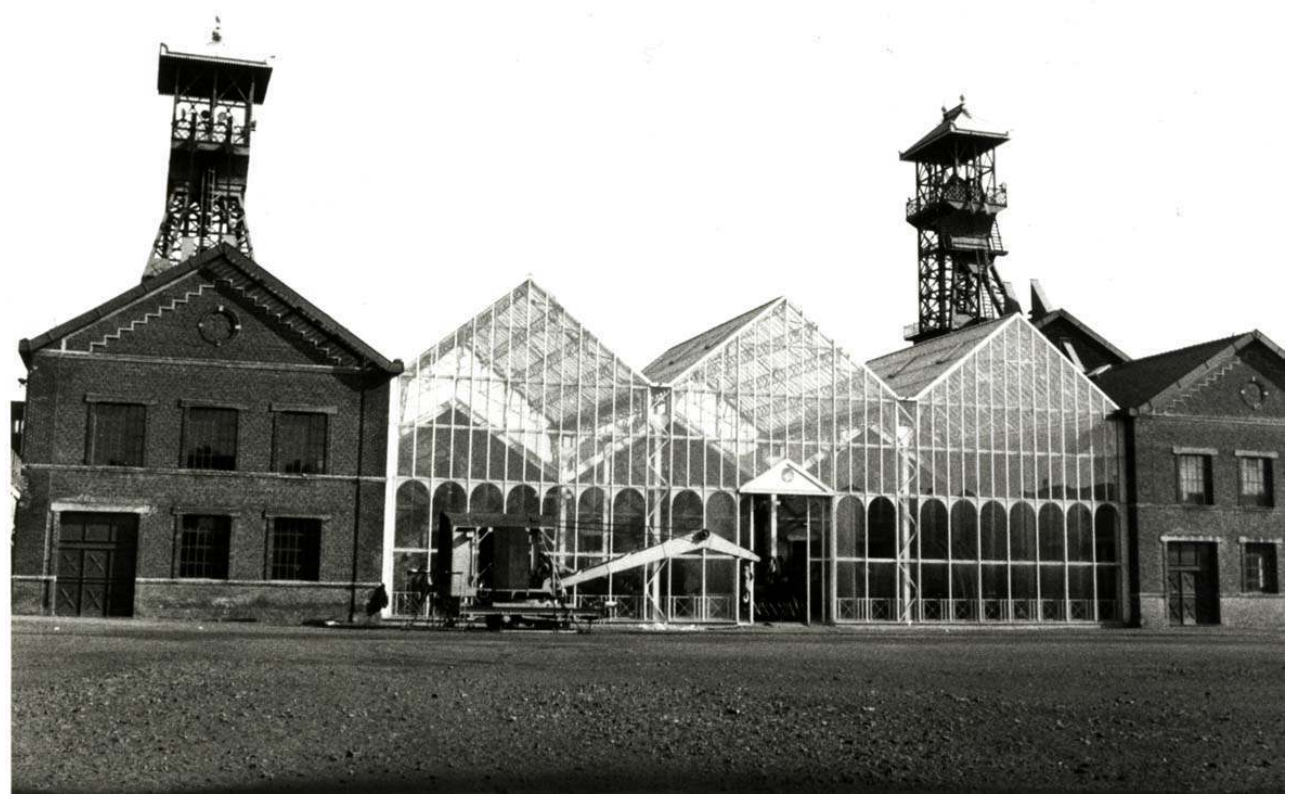

LEWARDE (NORD). VERRIÈRE DES MACHINES DU CENTRE HISTORIQUE MINIER.

(C) HBNPC, CENTRE HISTORIQUE MINIER, 1989.

10 C'est ce principe qui va être suivi durant toute la deuxième phase d'aménagement, celle engagée par l'Association du Centre historique minier après la disparition des Houillères. De 1999 à 2009, trois tranches de travaux de restructuration architecturale et muséographique sont menées. Le cahier des charges définit quatre piliers essentiels. Il consiste d'abord à sortir des bâtiments historiques de la fosse tous les services du Centre historique minier, c'est-à-dire l'administration, la billetterie, la boutique, les salles de réunion... Le second est de retrouver une plus grande authenticité du site en le rendant plus « lisible » : dans les années 1980, des ajouts de type architecture néo-romane avaient masqué l'architecture intérieure de la fosse ; leur suppression permet de retrouver les murs, les sols d'origine... (fig. 3). Le troisième pilier doit permettre d'assurer une cohérence de fonctionnement et un confort accru des dispositifs d'accès des publics. Au milieu des années 2000, la fréquentation du Centre atteint près de 150000 visiteurs par an. 
Figure 3

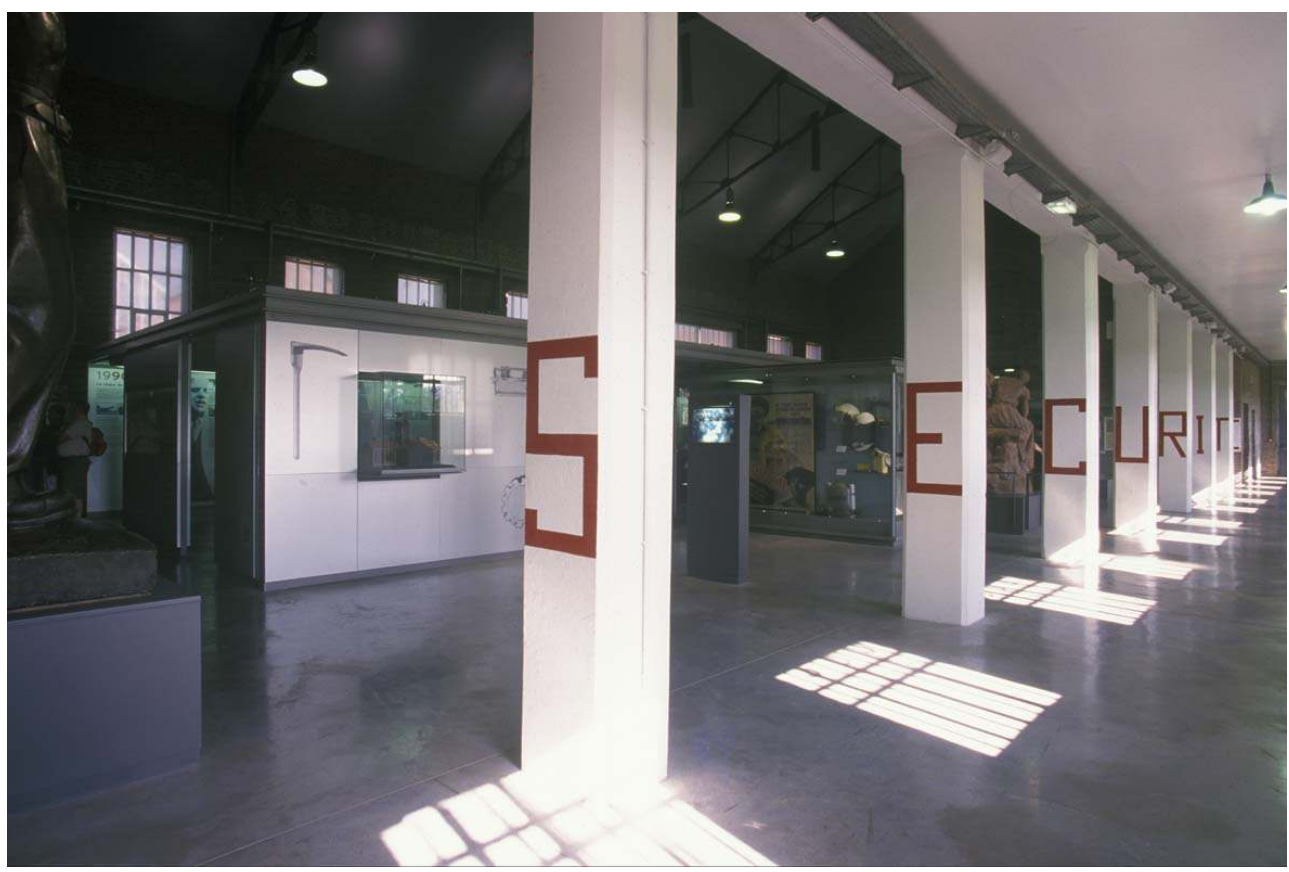

LEWARDE (NORD). GARAgE À VÉLOS.

PHOT. SUCHEYRE, DALHIETTE. (C) CENTRE HISTORIQUE MINIER, 2002.

Enfin, le quatrième pilier porte sur le redéploiement cohérent des collections dans des expositions permanentes présentées au cœur de la fosse Delloye. La ligne directrice définie par l'équipe du Centre pour les aménagements muséographiques consiste à proposer au public de découvrir non seulement un site minier, celui de la fosse Delloye, mais également des expositions scientifiques sur la géologie, l'histoire du bassin minier du Nord-Pas de Calais, la vie dans les cités minières ou les énergies. La découverte d'une fosse de mine et de son fonctionnement s'effectue sur les " pas des mineurs »: de l'entrée sur le lieu de travail par le "garage à vélos ", l'équipement des ouvriers dans la « salle de bains » puis en « lampisterie », leur trajet (la " passerelle du personnel », la « cage ») pour rejoindre les chantiers de travail (le "circuit minier " et 450 mètres de chantiers de creusement et d'abattage du charbon reconstitués), les métiers du jour (la «scierie », les salles des machines, l'« écurie »). Les lieux emblématiques de la fosse sont ainsi conservés et remis en état avec la plus grande authenticité. Les espaces moins spécifiques sont alors dédiés à des expositions permanentes: la salle de bains des ingénieurs et agents de maîtrise qui n'offre pas une spécificité minière est réinvestie pour présenter l'exposition d'histoire « Les trois âges de la mine».

\section{Le cheval à la mine, une exposition permanente au cœur de l'écurie de la fosse Delloye}

12 Cette exposition permanente «Le cheval et la mine » illustre la ligne suivie par l'équipe $\mathrm{du}$ Centre dans ses choix patrimoniaux, culturels et scientifiques : permettre au public de découvrir l'importance de l'histoire des chevaux à la mine et de visiter l'écurie de la fosse Delloye en respectant son authenticité. 


\section{Le cheval à la mine} le carreau des fosses pour le travail de roulage : le transport du matériel d'exploitation (outils, bois, explosifs...) et l'acheminement vers les voies de communication. Il assure également l'aérage des installations du fond. Un manège à chevaux actionne deux soufflets qui aspirent l'air vicié et refoulent de l'air frais dans les galeries. Mais c'est surtout comme force motrice que le cheval est utilisé jusqu'en 1800. L'exhaure (évacuation des eaux) et l'extraction s'effectuent exclusivement au moyen d'un manège à chevaux appelé « baritel » (fig. 4). Les chevaux actionnent un tambour vertical sur lequel s'enroule un câble qui passe sur deux grandes poulies, les «molettes». Quand une extrémité descend vers le fond, l'autre remonte un tonneau de charbon. Le sens des chevaux est ensuite inversé. À partir de 1802, la toute nouvelle machine à vapeur, nettement plus puissante, remplace progressivement la force de l'animal. Cependant, le cheval ne disparaît du jour que dans les années 1970.

\section{Figure 4}

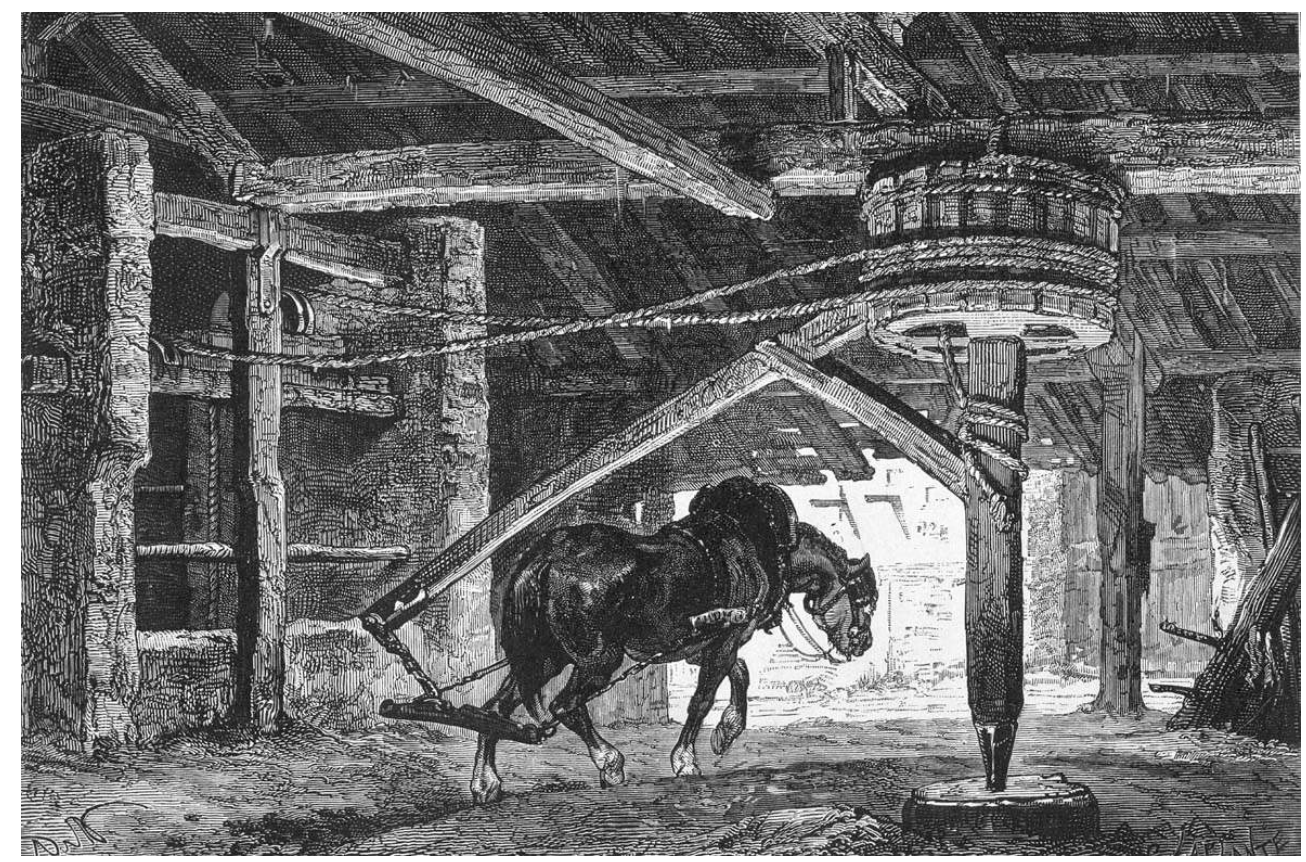

Le manège à chevaux, dans SIMONIN, Louis. La vie souterraine ou les mines et les mineurs, 1867. REPRO. CENTRE HISTORIQUe MINIER. (C) CENTRE HISTORIQUe MINIER, 2012.

C'est à partir de 1847 que le cheval est employé dans les galeries souterraines pour le roulage. Auparavant, les hercheurs, le plus souvent des jeunes apprentis, tiraient les esclittes (petits traîneaux surmontés d'une cuve en bois), puis poussaient les wagonnets. L'innovation décisive vient d'Angleterre et en 1847, des rails en fer sont posés au fond des galeries : ils permettent de faire circuler, depuis le pied de taille jusqu'à l'accrochage, des berlines dont la capacité atteint 500 litres. Si les berlines sont encore amenées par un homme jusqu'à la galerie principale, le train formé est alors tiré par un cheval. La force $\mathrm{du}$ cheval permet d'augmenter la production et le rend trois fois plus rentable que l'homme. Les chevaux tractent en moyenne 12 à 13 berlines par convoi (fig. 5). 
Figure 5

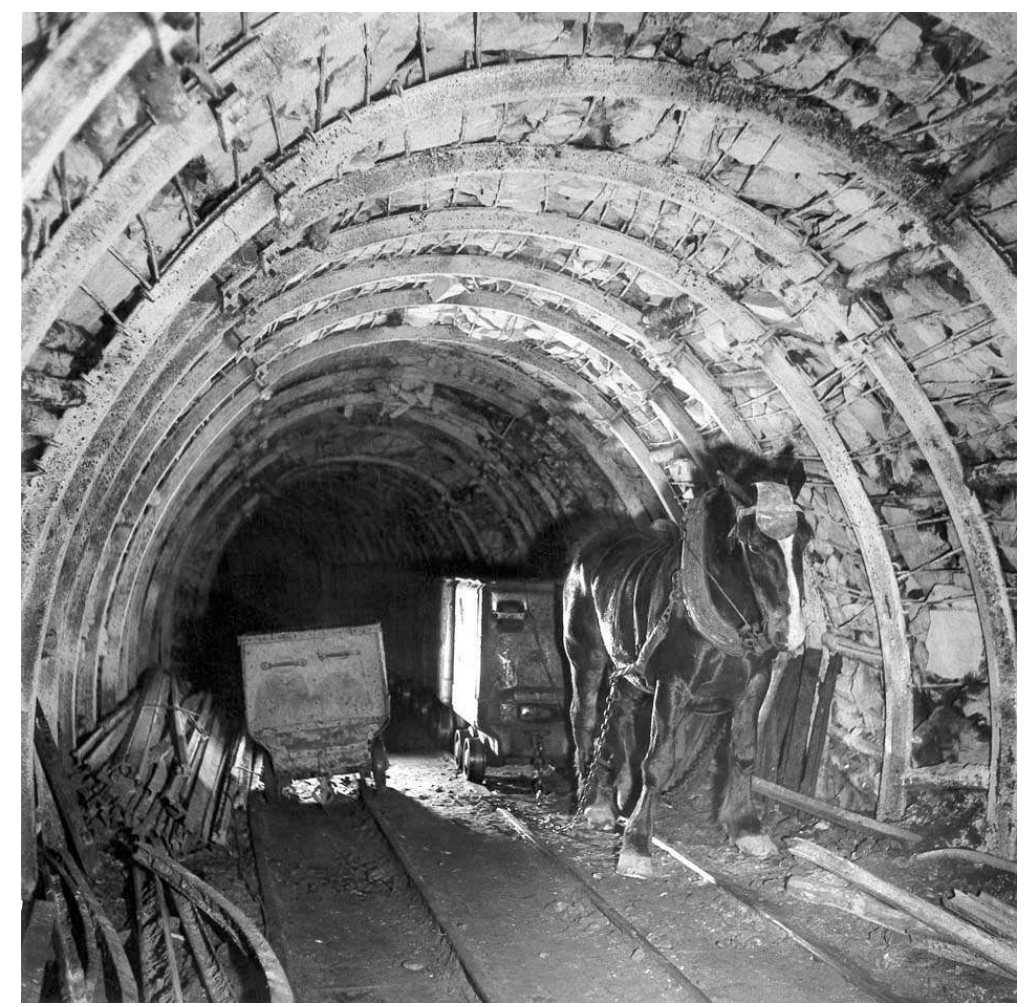

LEWARDE (NORD). CHEVAL TIRANT DES BERLINES AU FOND.

(C) HBNPC, CENTRE HISTORIQUE MINIER, 1963.

Le choix d'un cheval est dicté par le type de travail à fournir : tirer de lourdes charges sur de courtes distances avec des arrêts fréquents. Le choix se porte idéalement sur des chevaux dits " de gros trait lent ", calmes, musclés et de petite taille, vu la faible hauteur des galeries. Il doit aussi avoir de bons pieds, larges et sains. Dans la plupart des cas, ils sont sélectionnés par le vétérinaire attaché à la fosse et le responsable d'écurie. Les races les plus utilisées sont des chevaux lourds belges - brabançons et ardennais - et français traits du Nord, bretons ou auxois. Ces chevaux évoluent dans un environnement difficile, obscur, bruyant, parsemé d'obstacles et doivent donc être dociles et paisibles. C'est pour cette raison que le choix se porte essentiellement sur des chevaux hongres (castrés) de plus de cinq ans.

Les fosses sont équipées d'écuries au jour et au fond. Les écuries souterraines sont généralement situées dans des zones tempérées et bien aérées, près du puits de retour d'air et à l'écart des chantiers d'abattage. Les règlements stipulent en effet que «l'air des écuries ne doit traverser aucun chantier, ceci afin d'éviter la propagation d'un incendie qui prendrait naissance dans la paille ou le fourrage.» (fig. 6) Dans les années 1920-1940, la dimension des cages facilite la descente des chevaux mais permet surtout leur remonte quand des soins le nécessitent, en cas de grève et même pour des congés. Le personnel affecté aux chevaux est nombreux. Le conducteur a pour mission d'atteler, dételer, faire virer le cheval, et d'accrocher les wagonnets pour former un convoi. Le palefrenier assure le nettoyage des boxes, l'alimentation et le pansage de la cavalerie. Quant au maréchalferrant et au vétérinaire, ils descendent régulièrement pour soigner les animaux. 


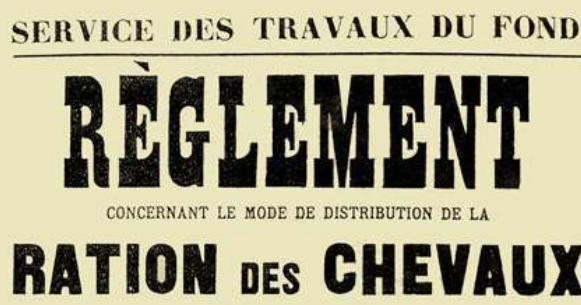

La ralliou sera distribure de la nanière suivante

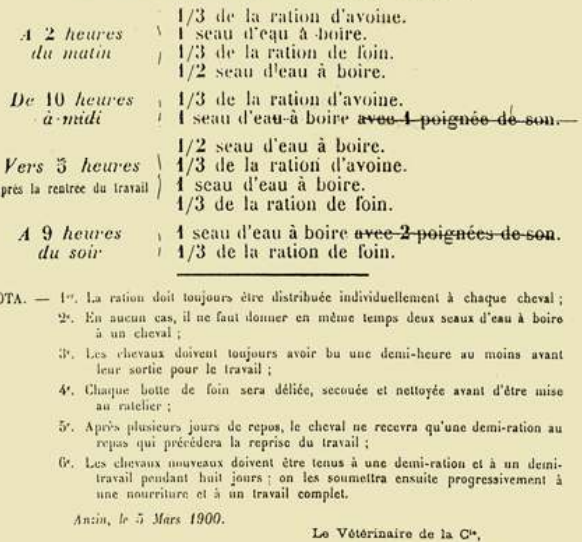

A. FIÉVET.

LEWARDE (NORD). SERVICE DES TRAVAUX DU FOND, RÈgLEMENT CONCERNANT LES RATIONS DES CHEVAUX, 1900.

REPRO CENTRE HISTORIQUE MINIER. @ CENTRE HISTORIQUE MINIER.

Dans certaines fosses, la mécanisation et l'apparition des locomotives Diesel puis électriques ont tendu à remplacer progressivement la force musculaire des chevaux. Cependant, le cheval reste longtemps le seul à pouvoir accéder à certaines voies. C'est en 1976 qu'est remonté Bambino, le dernier cheval de mine du bassin houiller du Nord-Pasde-Calais.

\section{Un lieu, des collections}

L'écurie du jour de la fosse Delloye fut installée en 1937 sous le clichage du puits nº 1, là où transitaient les berlines de charbon. Elle y accueillait les chevaux affectés au jour sur le carreau mais aussi ceux remontés du fond en raison de maladie, de blessure ou d'arrêt prolongé du personnel (congés, grèves...). Du fait de sa date de construction, les dimensions des cages de la fosse facilitent la descente et la remonte des chevaux. D'une superficie de $260 \mathrm{~m}^{2}$, l'écurie est équipée de stalles parallèles et de mangeoires façonnées dans le mur. Pour faciliter leur nettoyage, les sols sont bétonnés ou pavés, aménagés avec une rigole à urine légèrement pentue (fig. 7). 


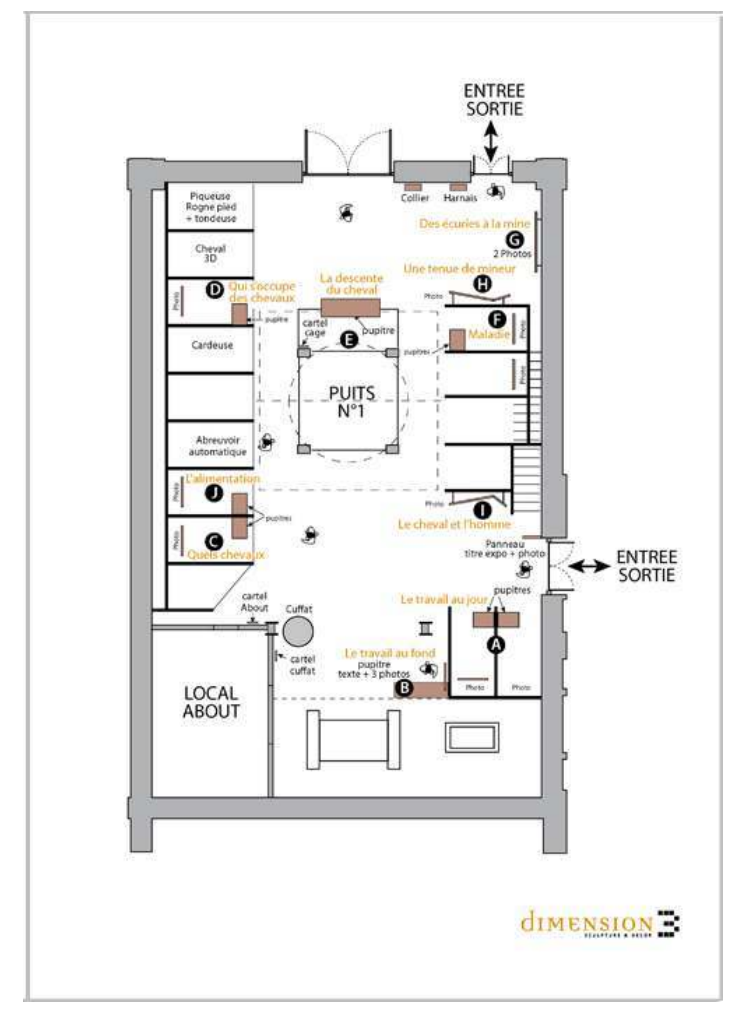

LEWARDE (NORD). PLAN D'IMPLANTATION DE L'EXPOSITION « LE CHEVAL ET LA MINE ». DIMENSION 3. (C) CENTRE HISTORIQUE MINIER, 2009.

Parmi les objectifs de 1976, les Houillères du bassin du Nord-Pas-de-Calais avaient fixé celui de collecter et conserver des matériels, des outils, des objets et des archives relatifs à la mine et à la culture minière. De 1976 à 1993, des équipes se sont mobilisées afin de récupérer ces différents matériaux au fur et à mesure des fermetures des fosses et des services sur l'ensemble du territoire du bassin minier du Nord-Pas de Calais. De même, les collectes lancées par l'équipe du Centre à partir de 1993 et les acquisitions ont permis de compléter les collections et les fonds d'archives.

Le Centre conserve ainsi dans ses collections de nombreux objets et matériels relatifs aux chevaux et à leur travail : harnais en cuir, colliers, piqueuse (machine à coudre servant au bourrelier pour confectionner les harnais), rogne-pieds, seringue, cardeuse, tondeuse... Les archives sont, quant à elles, très importantes et variées: livrets d'ouvrier des chevaux, règlements et rapports du service vétérinaire (fig. 8), affiches de consignes, rapports d'accidents, plans d'écuries souterraines ou du jour. Les fonds iconographiques conservent des séries de cartes postales du début du $\mathrm{xx}^{\mathrm{e}}$ siècle illustrant les descentes des chevaux, les différents métiers (maréchal-ferrant, palefrenier...), le roulage souterrain. Les campagnes photographiques menées par le service des relations publiques des HBNPC dans les années 1950 à 1970 montrent aussi les liens entre le cheval et le mineur. Enfin, parmi les 7000 ouvrages de la bibliothèque, on peut trouver, outre les principaux livres consacrés aux chevaux à la mine, des cours d'exploitation technique dans lesquels sont développés l'évolution des techniques et notamment le roulage mais aussi des thèses de vétérinaires. 


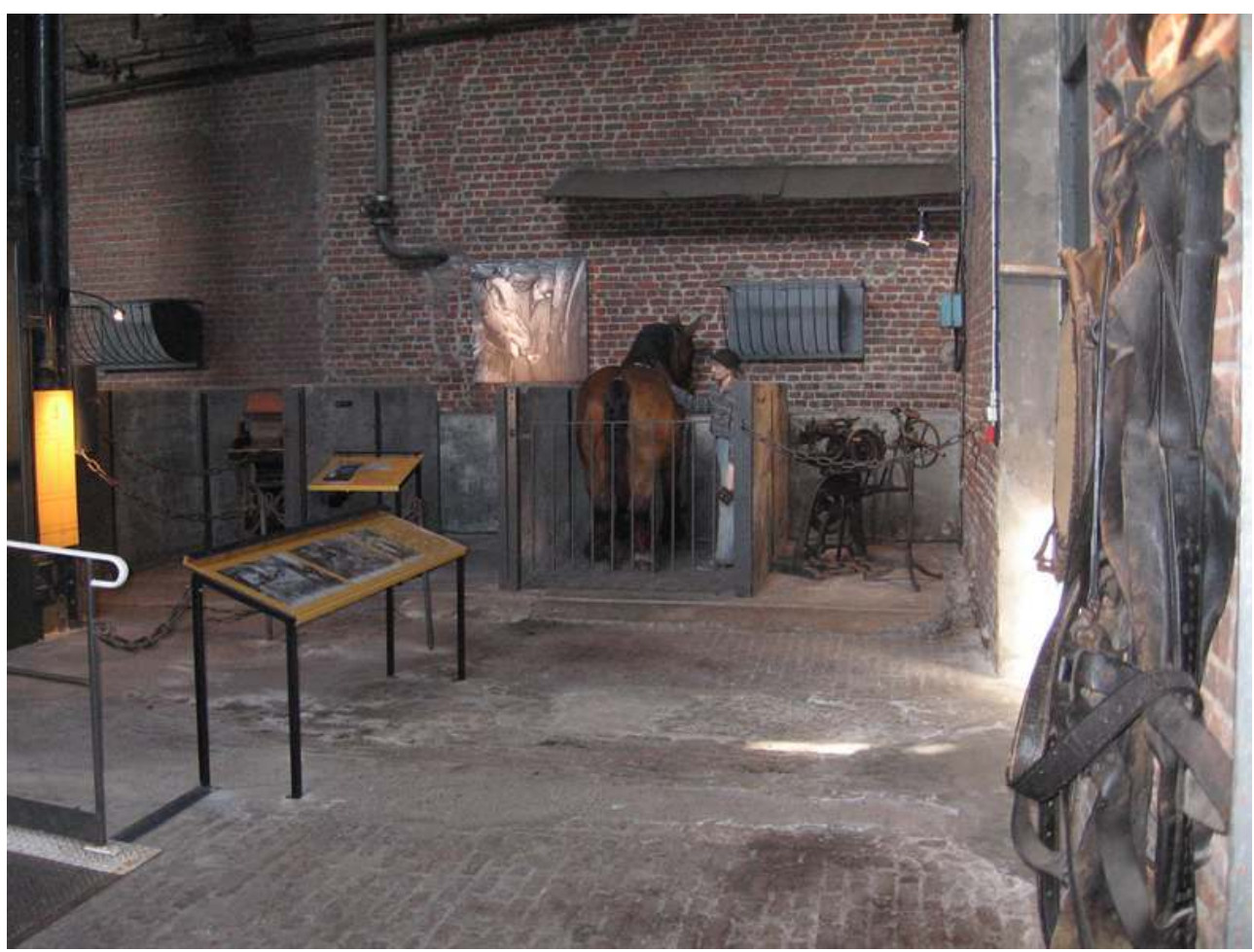

LEWARDE (NORD). VUE DE L'ÉCURIE DE LA FOSSE DELLOYE.

(C) CENTRE HISTORIQUE MINIER, 2010.

\section{Les choix muséographiques}

21 L'écurie, comme d'autres espaces de travail de la fosse, a fait l'objet au début des années 1990 d'une remise en état. Une première exposition présentait une reconstitution fidèle de l'écurie de Delloye dans les années 1930 et des modules concernant les conditions de vie et de travail du cheval dans la mine. Deux mannequins de cheval et de palefrenier étaient alors installés dans une stalle. La troisième tranche de travaux, en 2009, a permis d'intervenir à nouveau dans cet espace. L'authenticité de l'écurie a été respectée, tant au niveau des structures que des sols; l'accessibilité y est pourtant renforcée par la construction d'une passerelle d'accès pour les personnes à mobilité réduite.

La muséographie intervient sur deux types de contenu. Le premier est destiné à la découverte de l'écurie par le public. Pour cela, des cartels sont placés pour expliquer et décrire l'ensemble du lieu et des machines présentées : l'écurie de la fosse Delloye, le puits $n^{\circ} 1$, la cage d'extraction, le treuil mais aussi la berline et le cuffat. Le second contenu est bien sûr consacré au cheval. Différentes thématiques sont développées : le travail au jour, le travail au fond, les types de chevaux, l'alimentation, les personnels affectés, les maladies, la descente au fond, les écuries au jour et au fond, la tenue de mineur du cheval et les relations entre homme et cheval.

Le cahier des charges remis à l'équipe de scénographes précise que la muséographie doit être légère, discrète, facilement démontable tout en respectant les règles d'accessibilité pour les différents publics (taille des caractères, contraste des couleurs, hauteur 
appropriée des éléments exposés...) Ce sont deux types de supports qui ont été créés pour le contenu: deux modules de textes illustrés à chacune des entrées de l'écurie et des cartels sur pupitres dans les stalles. Ces grands cartels s'appuient aussi sur des reproductions de documents d'archives, tels que des rapports de vétérinaires, des consignes... Dans certaines stalles, les mangeoires sont remplacées par des photographies grand format. Enfin, une bande sonore, composée de hennissements et de bruits des outils du maréchal-ferrant, permet aux visiteurs de plonger dans l'ambiance de l'écurie. Cette sonorisation est aussi un appel pour le public qui sort de la boutique située en face de l'entrée de l'écurie.

\section{NOTES}

1. - Virginie Debrabant est directrice des Archives et du centre de Ressources documentaires depuis 2000. Coordinatrice du conseil scientifique du musée, elle est commissaire des expositions temporaires et assure l'ensemble des publications ainsi que l'organisation des séminaires et des colloques scientifiques du Centre historique minier.

\section{RÉSUMÉS}

Le Centre historique minier est implanté sur le site de la fosse Delloye, qui a fonctionné de 1931 à 1971. Cette fosse de mine dispose de toutes ses infrastructures, notamment son écurie du jour où le public peut découvrir l'exposition permanente « Le cheval et la mine ». Au XVIII ${ }^{\mathrm{e}}$ siècle, la seule énergie disponible dans les mines du Nord-Pas-de-Calais était la force musculaire, celle des hommes et des chevaux. Au jour, les chevaux de trait actionnaient des sortes de manèges, les baritels, qui servaient à descendre et remonter les produits d'extraction. Au milieu du XIx ${ }^{\mathrm{e}}$ siècle, dès que la taille des puits le permit, les chevaux descendirent eux aussi au fond pour tirer les berlines jusqu'à l'accrochage. L'arrivée de nouvelles énergies, la vapeur, l'air comprimé, puis l'électricité, n'exclut cependant pas le travail du cheval au fond des mines et le dernier cheval remonta en 1976. Plus qu'un simple animal de travail, le cheval de la mine était considéré comme un ouvrier à part entière, mais c'était aussi un vrai compagnon de travail, très apprécié du mineur, dont il partageait le dur labeur.

The horse from the mine to the museum, the example of Lewarde historic mining centre. The Lewarde mining history centre is established on the site of the Delloye colliery which operated from 1931 to 1971. This pit still features its former infrastructures, such as its above-ground stable, where the public can discover the permanent exhibition entitled 'Horses and Mining'. In the eighteenth century, the only source of energy available down the mines of the Nord-Pas-deCalais region was the muscular power, of men and horses. Above ground, draft horses drove winding gins, which could lower the men down and raise the extracted coal up. In the middle of 
the nineteenth century, when the size of the shafts made it possible, horses were sent underground to haul the mine wagons at the pit bottom. The arrival of new energy sources, steam, compressed air, then electricity, did not put an end to the work carried out by the horses underground and the last horse was brought to the surface only in 1976. More than a working animal, the horse was considered as a fully-fledged worker, but also as a real co-worker, very popular amongst the miners, with whom it shared the hard work.

INDEX

Mots-clés : cheval, musée, mine de charbon, cheval de mine, histoire de la mine, Centre historique minier de Lewarde

Keywords : coal mine, mining history, Lewarde Centre Historique Minier, horses, pit horses

\section{AUTEUR}

\section{VIRGINIE DEBRABANT}

directrice des Archives et du centre de Ressources documentaires du Centre historique minier vdebrabant@chm-lewarde.com 